\title{
Reciclaje de Residuos de Construcción y Demolición (RC\&D) Generados en la Universidad del Valle Sede Meléndez para la Fabricación de Adoquines
}

\author{
Santiago Ceballos-Medina*; Diana Carolina González-Rincón; Julián David Sánchez \\ Escuela de Ingeniería de Materiales, Universidad del Valle, Calle 13 \#100-00, Cali, Colombia. \\ *santiago.ceballos@correounivalle.edu.co
}

\begin{abstract}
Resumen
Se presenta una propuesta de aprovechamiento y reciclaje de los residuos de construcción y demolición RC\&D para la preparación de agregados de construcción como insumo en la fabricación de adoquines a partir de los residuos generados en la Escuela de Ingeniería de Materiales (EIMAT) de la Universidad del Valle sede Meléndez. Para ello, se identificaron los puntos de concentración de escombros en la Universidad junto con su clasificación y selección, así como el proceso de preparación de agregados y elaboración de adoquines. A estos últimos se les realizaron ensayos con el fin de evaluar su viabilidad al compararlos con los adoquines tradicionales, tales como pruebas de: absorción de agua, densidad y resistencia a la flexotracción (módulo de rotura), obteniendo respectivamente los siguientes resultados: $3,52 \% ; 1608,21 \mathrm{~kg} \mathrm{~m}^{-3} ; 3,5 \mathrm{MPa}$ (7 días). Se concluye que los agregados obtenidos por medio de la trituración de escombros (morteros, ladrillos y concretos) poseen un buen desempeño en adoquines.
\end{abstract}

Palabras clave: RC\&D; Agregados; Reciclaje; Adoquines; Escombros. 


\title{
Construction and Demolition Waste (C\&DW) Recycling Generated at the Universidad del Valle (Meléndez) for the Manufacture of Paving Stones
}

\begin{abstract}
The main objective of this paper is to show a proposal about the use and recycling of construction and demolition wastes (C\&DW) for the preparation of aggregates as a raw material in the production of paving stones obtained from Escuela de Ingeniería de Materiales (EIMAT), Universidad del Valle sede Meléndez wastes. Construction wastes concentration points at University are shown along with their classification and selection. The process of production of aggregates and paving stone is also present. The characterization of the paving stones has be done using the following tests: water absorption, density and flexural strength and compare with those of normal aggregates; obtaining respectively the following results: $3,52 \% ; 1608,21 \mathrm{~kg} \mathrm{~m}^{-3} ; 3,5 \mathrm{MPa}$ (7 days). The results of the tested properties show that the aggregates obtained by crushing C\&DW (mortars, bricks and concrete) have a good performance in paving stones.
\end{abstract}

Keywords: C\&DW; Aggregates; Recycling; Paving Stone.

\section{Reciclagem de Resíduos de Construção e Demolição (C\&DW) Gerada na Universidad del Valle (Meléndez) para Fabricação de Pedras de Pavimentação}

\begin{abstract}
Resumo
O objetivo principal deste artigo é apresentar uma proposta de aproveitamento e reciclagem de resíduos de construção e demolição (RCD) para a preparação de agregados como matéria-prima na produção de pedras de pavimentação obtidas na Escola de Ingeniería de Materiales (EIMAT), Resíduos da Universidad del Valle sede Meléndez. Os pontos de concentração de resíduos de construção na Universidade são mostrados junto com sua classificação e seleção. O processo de produção de agregados e pedra de pavimentação também está presente. A caracterização das pedras do pavimento foi feita através dos seguintes ensaios: absorção de água, densidade e resistência à flexão e comparação com agregados normais. obtendo respectivamente os seguintes resultados: $3,52 \% ; 1608,21 \mathrm{~kg} \mathrm{~m}^{-3} ; 3,5 \mathrm{Mpa}$ (7 dias). Os resultados das propriedades testadas mostram que os agregados obtidos por britagem de C\&DW (argamassas, tijolos e concreto) apresentam um bom desempenho em pedras de pavimentação.
\end{abstract}

Palavras-chave: RCD; Agregados; Reciclagem; Pedra de pavimentação. 


\section{Introducción}

Uno de los principales problemas ambientales causados por el sector de la construcción son los grandes volúmenes de residuos de construcción y demolición (RC\&D) que se generan debido al incremento constante de obras asociadas a este sector. En Colombia, el sector de la construcción es uno de los más relevantes y con mayor demanda; este se encuentra asociado a factores como: el desarrollo socioeconómico, crecimiento poblacional, ordenamiento territorial (desarrollo de infraestructura física), entre otros. Como resultado de ello se generan cantidades considerables de RC\&D en el país [1].

Como se cita en Acevedo [2], dichos residuos presentan distintos inconvenientes que van desde su generación, transporte y disposición final. Se estima que, durante la etapa de construcción, el $10 \%$ de los materiales utilizados se convierten en residuos, y durante las operaciones de demolición y renovación se pueden producir hasta diez veces más residuos. Además, de acuerdo con la Unión Internacional para la Conservación de la Naturaleza (UICN) [3], debido al gran volumen que estos ocupan, existe una problemática asociada a la disposición final en los rellenos sanitarios pues provocan la disminución de vida útil de estos lugares.

Particularmente, en la ciudad de Cali se generan en promedio $2500 \mathrm{~m}^{3}$ diarios de RC\&D. De este volumen, cerca de $76,6 \%\left(1900 \mathrm{~m}^{3}\right)$ es aportado por las constructoras y obras públicas en lo que se denomina "la generación formal" y 23,4 \% (580 $\mathrm{m}^{3}$ ) restante son aportados por construcciones y remodelaciones particulares, y el llamado "sector informal" como lo menciona Salazar en [1]. En el Plan de Gestión Integral de Residuos Sólidos (PGIRS) [4] de la ciudad se establece que, del total de escombros generados, el $60 \%$ corresponden a materiales aprovechables, mientras que el 40 $\%$ restante serán suelos, material de descapote y excedentes de excavación que serán dispuestos para nivelación de terrenos, recuperación geomorfológica y/o su disposición final [5]. Debido a los RC\&D pueden aparecer sitios no autorizados para el arrojo de escombros, creando focos de contaminación en el municipio. Los RC\&D que se encuentran en estas estaciones pueden no encontrarse en las mejores condiciones debido a que son llevados y combinados con residuos orgánicos $[4,6]$.
Por otro lado, la Universidad del Valle no se encuentra muy alejada de estas problemáticas pues cuenta con las escuelas de Ingeniería de Materiales e Ingeniería Civil las cuales constantemente desarrollan prácticas, laboratorios e investigaciones relacionadas con los materiales cementicios y de construcción. Finalizadas estas actividades necesarias para la formación de los estudiantes y la investigación, se genera una cantidad importante de residuos, los cuales son depositados en las escombreras de cada una de las escuelas, como se observa en la figura 1. Estos residuos de no ser aprovechados como reciclaje terminarán contribuyendo de manera negativa a la problemática actual de la ciudad.

Por lo anterior, el reciclaje de este tipo de residuos se presenta como una gran opción para mitigar el impacto que estos ejercen en el medio ambiente de ahí la importancia del estudio de nuevas alternativas para su mejor aprovechamiento. Según Salazar [1], estas actividad trae los siguientes beneficios:

- Reducir focos de contaminación y problemas de salubridad relacionados con este (suelo y aire contaminado)

- Reducción considerable en los costos de manejo y disposición final por parte de las autoridades municipales encargadas, además de una reducción en los gastos asociados a la limpieza por el vertimiento inapropiado de estos residuos alrededor de toda la ciudad.

- Disminución de botaderos de escombros no autorizados, reduciendo los riesgos ambientales y evitando la desvalorización de los predios cercanos a estos lugares.

- Producción de agregados reciclados de concreto (ARC) tanto gruesos como finos para la producción de mezclas de concreto y mortero, bases y sub-bases granulares y elementos prefabricados.

- Reducción en la demanda y extracción de materias primas naturales como grava y arena para el uso en el sector de la construcción.

El estudio experimental sobre estos procesos ha ido creciendo paulatinamente. Un estudio de vigilancia realizado a través de la base de datos Scopus revela cómo, durante los últimos 10 años, el número de artículos científicos y técnicos que contengan como palabra clave "CDW aggregates" ha ido en aumento, como lo muestra la figura 
2. Los países que lideran esta lista son China, Brasil y España, los cuales tienen una fuerte industria de construcción; además, presentan una gran inversión en esta área de las ciencias e investigación técnica.
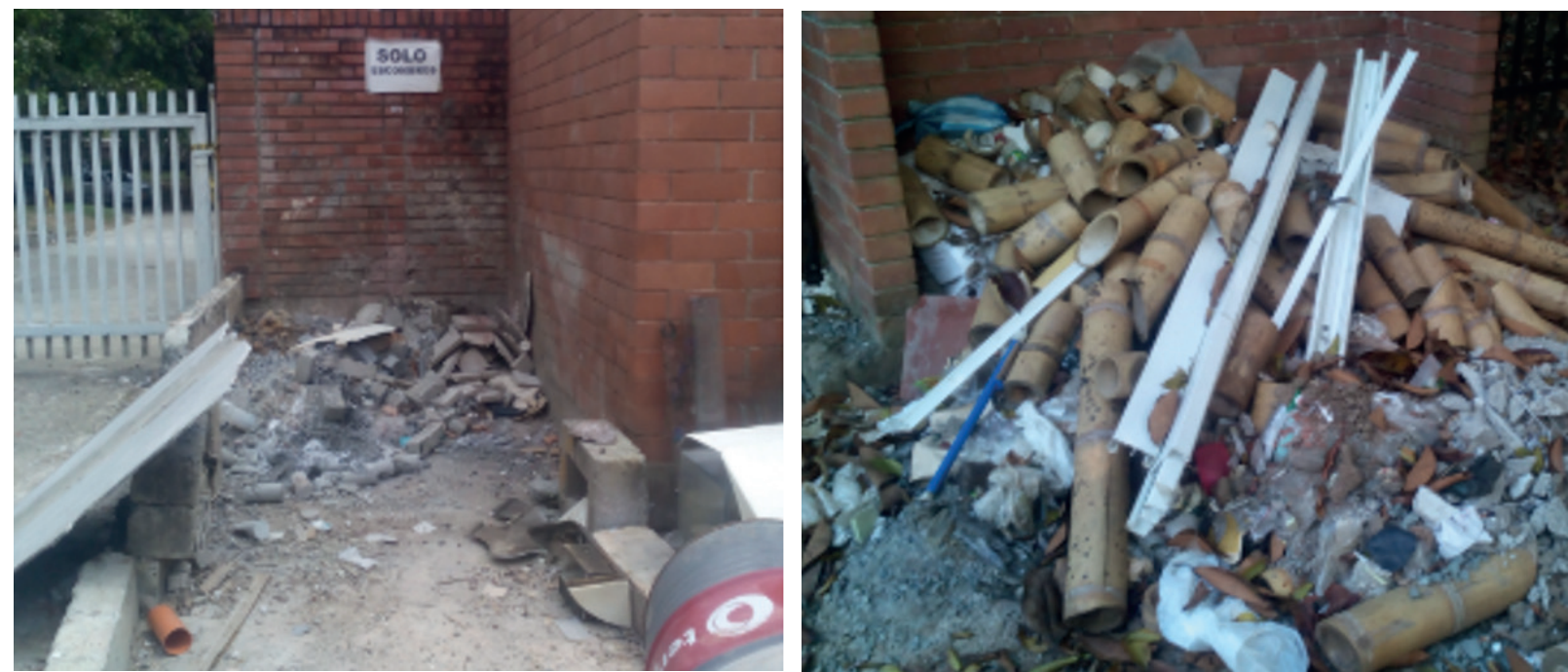

Figura 1. Escombreras de Escuela de Ingeniería de Materiales (izquierda) y Escuela de Ingeniería Civil (derecha).

Se estima que China produjo alrededor de 2.360 toneladas de RC\&D en la última década $\mathrm{y}$, actualmente, la tasa de reciclaje promedio para esta clase de residuos es tan solo del $5 \%$. Por esta razón, China lidera el número de publicaciones en torno a la investigación técnica en busca de alternativas para el uso de los RC\&D. Un ejemplo de esto se aprecia en el trabajo de Zou y colaboradores [7], quienes estudiaron la incorporación de agregados de concreto reciclado como sustituto de agregados naturales en asfalto espumados. Investigaron los distintos factores que influyen en la calidad del asfalto. Ellos concluyeron que, es posible reemplazar el $100 \%$ de los agregados naturales con agregados a partir de RC\&D cumpliendo con los requerimientos técnicos necesarios para la fabricación de la base y la subbase del pavimento asfáltico. Otro buen ejemplo de la versatilidad del uso de RC\&D se encuentra en el trabajo de Beja y colaboradores [8], donde se estudia la viabilidad de utilizar el reciclaje de RC\&D como agregados en pavimentos de alto volumen de tráfico pesado para las vías de Brasil. Encontraron que los agregados de RC\&D pueden usarse con éxito como material de sub-base de pavimento bajo carga de tráfico pesado y que la rigidez del agregado RC\&D aumentó con el tiempo. A nivel nacional, la cifra de publicaciones es demasiado baja (1 artículo en los últimos 10 años), lo que refleja la necesidad de fortalecer las capacidades nacionales en este campo que resulta ser pertinente en la agenda ambiental actual; también representa un nicho de mercado con gran potencial al ser un país en vía de desarrollo que podría presentar una fuerte contribución para la disminución de la brecha científica y tecnológica de Colombia frente a otros países de la región y el mundo en temas de innovación y desarrollo.

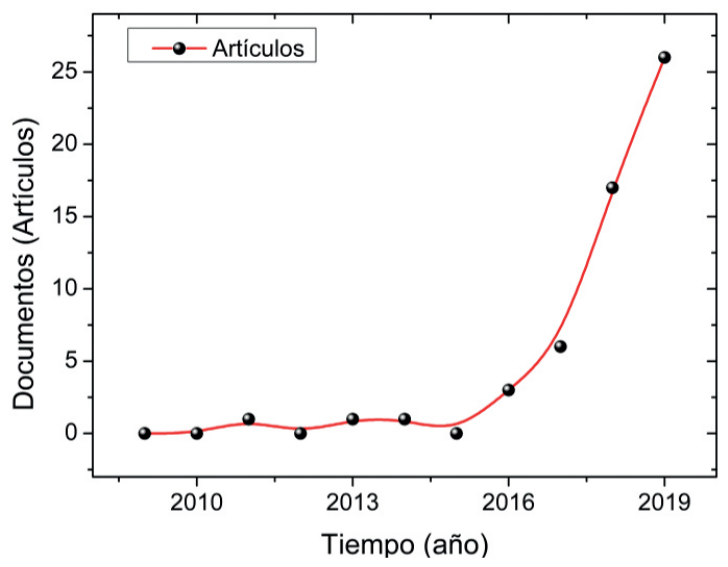

Figura 2. Representación gráfica del resultado de la búsqueda de vigilancia para artículos publicados en los últimos 10 años (2009-2019). 
En este artículo se busca estudiar el uso de los agregado reciclados provenientes de los RC\&D de la escuela de Ingeniería de Materiales de la Universidad del Valle para la fabricación de adoquines con el fin de evaluar su viabilidad como alternativa para los elaborados tradicionalmente en la industria. Adicionalmente, debido a las grandes expectativas que genera la implementación de esta solución en torno al reciclaje

\section{Detalles Experimentales}

\section{Agregados}

Para la adecuada selección de los RC\&D en la preparación de agregados, se realizó un diagnóstico en los puntos de concentración de la Universidad del Valle sede Meléndez, en donde se encontraron los siguientes tipos de escombros:

- Mortero.

- Ladrillo.

- Concreto.

- Vidrio.

- Madera de guadua.

- Acero estructural.

Se optó por hacer uso de los residuos generados en la Escuela de Ingeniería de Materiales por los siguientes motivos:

La cercanía de estos materiales al equipo de trituración y a la máquina bloquera. La presencia de mortero, ladrillo y concreto (ver figura 3) para la obtención de los agregados finos y gruesos necesarios para realizar los adoquines.
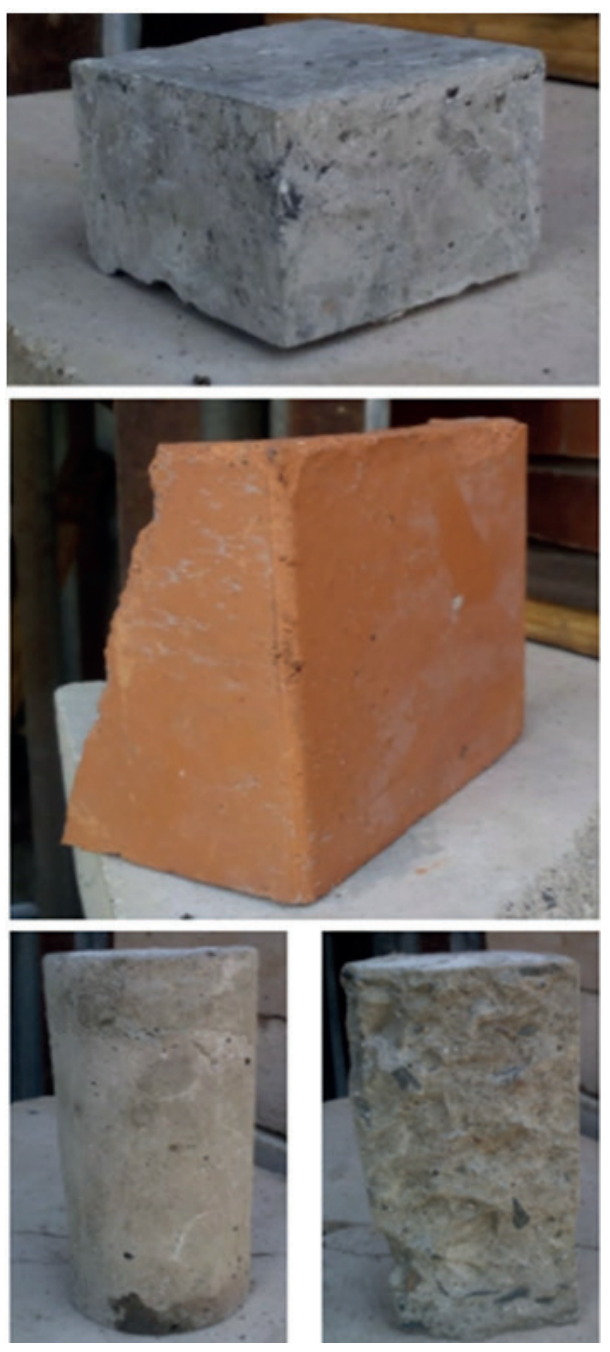

Figura 3. Escombros de mortero (arriba), ladrillo (en medio) y concreto (abajo) que fueron usados. 
Trituración de los RC\&D: Los residuos fueron sometidos a una trituración mecánica por medio de una trituradora de mandíbulas hasta ser reducidos a tamaños no mayores a aproximadamente $3 / 8$ " $(9,525 \mathrm{~mm})$, obteniendo de esta forma agregados finos y gruesos. Para garantizar el tamaño máximo de partícula, se realizó un cribado manual, haciendo pasar los agregados por un tamiz de 3/8 de pulgada de abertura, obteniendo así un gradiente granulométrico en los agregados. Los agregados obtenidos se usaron como materia prima para la fabricación.

\section{Adoquines}

\section{Materiales empleados}

- Cemento Portland Tipo I, marca Topex

- Agregados reciclados (escombros de mortero, ladrillo y concreto), tamaño máximo $3 / 8$ de pulgada

Diseño de la mezcla: el diseño se realizó por peso; las proporciones se encuentran en la tabla 1.

Tabla 1. Diseño de la Mezcla

\begin{tabular}{ccc}
\hline Cemento (kg) & Agregados $\mathbf{( k g )}$ & Agua $\mathbf{( k g )}$ \\
\hline 1,00 & 4,00 & 0,45 \\
\hline
\end{tabular}

Elaboración de adoquines: se mezclaron manualmente los adoquines en las proporciones establecidas $y$, posteriormente, se fabricaron 7 adoquines empleando la máquina bloquera la cual realiza un adoquín tipo 2 o "hueso de perro" según la NTC 2017 [9] (ver figura 4).

\section{Curado y Fraguado}

Para el curado de los adoquines, estos se sumergieron en un tanque de agua durante siete días, para garantizar una mejor reacción del cementante y así obtener unas mejores propiedades mecánicas.

\section{Ensayos}

\section{Absorción de Agua y Densidad}

Los siete adoquines fueron sumergidos, durante una semana dentro de un recipiente lleno de agua; luego cada muestra se retiró del agua y se pesó inmediatamente para obtener su masa saturada (húmeda) (Mh). Posteriormente se dejaron secar los adoquines a temperatura ambiente durante tres días y se obtuvo la masa seca de cada uno de ellos (Ms).

La absorción de agua (Aa) del total de la masadel espécimen se calculó para cada muestra mediante la siguiente ecuación:

$$
\operatorname{Absorción~}(A a)[\%]=\frac{M_{h}-M_{S}}{M_{S}} * 100
$$

\section{Resistencia a la Flexotracción}

El ensayo de flexotracción se realizó en la Escuela de Ingeniería de Materiales usando una máquina universal de ensayos Tinius Olsen H50KS, siguiendo los parámetros de la norma NTC 2017 como se muestra en la figura 5 .
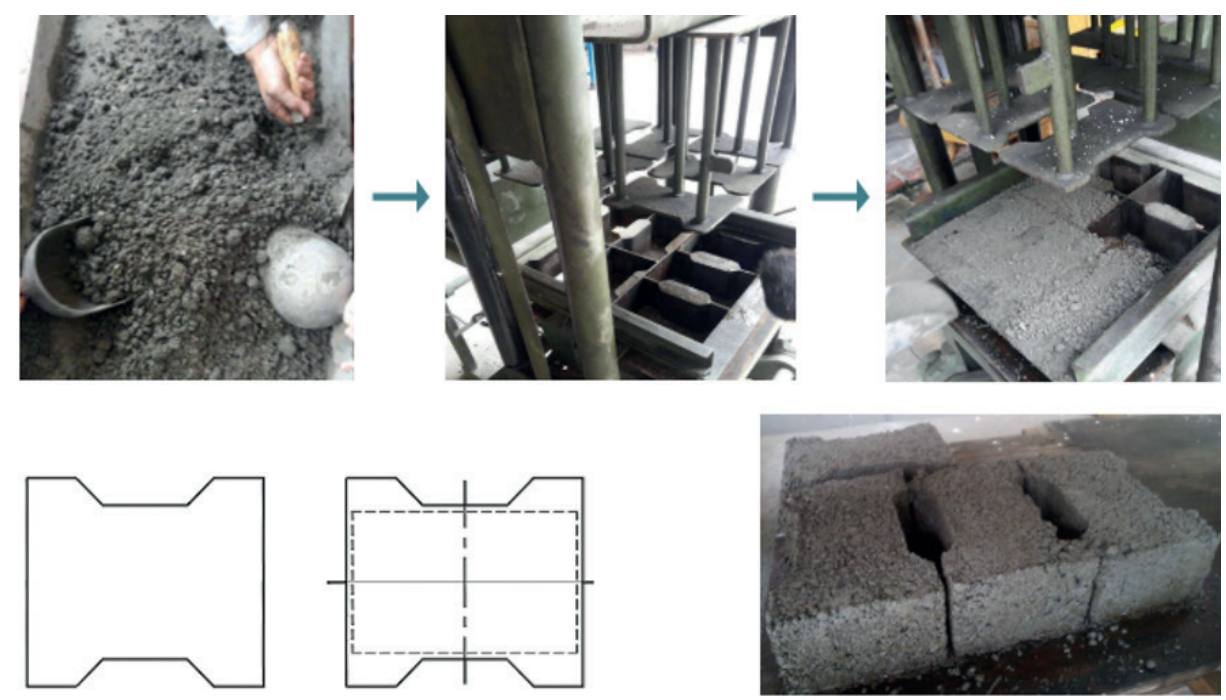

Figura 4. Secuencia Elaboración de Adoquines. 

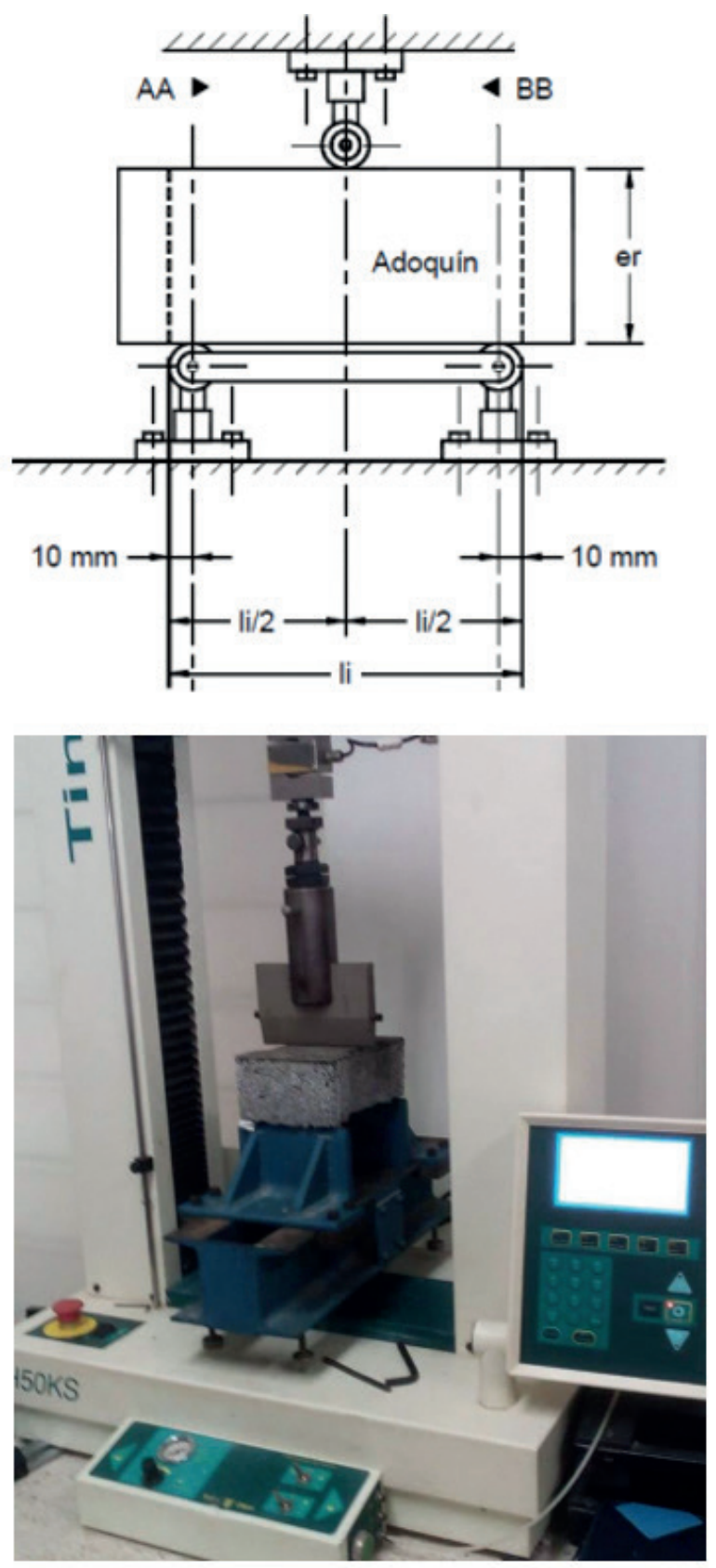

Figura 5. Montaje empleado para la realización del ensayo.

Para cada adoquín, se calcula el módulo de rotura (Mr) según la fórmula (2) dada por la NTC 2017 [9]. Donde "li" es la longitud del rectángulo inscrito, " $a r$ " el ancho real del espécimen, "ai" el ancho del rectángulo inscrito y "er" el espesor real del espécimen, todo en $\mathrm{mm}$.

$$
M r=\frac{3 C_{\max }(l i-20)}{(a r+a i) e r^{2}}
$$

\section{Resultados}

\section{Absorción de agua}

Los valores de Mh, Ms y el \% de absorción calculado con (1) se registraron en la tabla 2.

Tabla 2. Absorción de Agua.

\begin{tabular}{cccc}
\hline Muestra & $\mathbf{M}_{\mathbf{h}} \mathbf{( k g )}$ & $\mathbf{M}_{\mathbf{s}} \mathbf{( k g )}$ & $\mathbf{A}_{\mathbf{a}}(\mathbf{\%})$ \\
\hline 1 & 3,86 & 3,76 & 2,66 \\
2 & 3,44 & 3,36 & 2,38 \\
3 & 3,46 & 3,40 & 1,76 \\
4 & 3,52 & 3,40 & 3,53 \\
5 & 3,48 & 3,32 & 4,82 \\
6 & 3,36 & 3,24 & 3,70 \\
7 & 3,30 & 3,12 & 5,77 \\
\hline Promedio & $\mathbf{3 , 4 9}$ & $\mathbf{3 , 3 7}$ & $\mathbf{3 , 5 2}$ \\
D. Estándar & $\mathbf{0 , 1 8}$ & $\mathbf{0 , 2 0}$ & $\mathbf{1 , 4 1}$ \\
\hline
\end{tabular}

\section{Densidad}

El cálculo de la densidad fue realizado midiendo las aristas del adoquín tipo hueso, hallando así un volumen promedio de $0,002170116 \mathrm{~m}^{3} \mathrm{y}$, al relacionarse este valor con el peso seco promedio (ver tabla 2) de los adoquines, se obtuvo que la densidad promedio correspondiente a los adoquines de este trabajo es de: $1608,21 \mathrm{kgm}^{-3}$.

\section{Resistencia a la flexotracción}

Con el ensayo de flexotracción se logró determinar el esfuerzo máximo al cual los adoquines presentan una rotura en su estructura (ver figura 6 ). Se registraron los valores obtenidos (ver tabla 3) para posteriormente comparar estos valores con los adoquines encontrados en la industria (dictaminados por la norma NTC 2017) y se realizó el gráfico de esfuerzo-deflexión (ver figura 7).

Tabla 3. Módulo de rotura de los adoquines con agregados de escombro reciclado.

\begin{tabular}{ccc}
\hline Muestra & $\begin{array}{c}\text { Módulo de } \\
\text { rotura a 7 días } \\
\text { (MPa) }\end{array}$ & $\begin{array}{c}\text { Módulo de } \\
\text { rotura a 28* días } \\
\text { (MPa) }\end{array}$ \\
\hline 1 & 3,1 & 4,8 \\
2 & 3,6 & 5,5 \\
3 & 3,9 & 6,0 \\
\hline Promedio & 3,5 & 5,4 \\
D. Estándar & 0,4 & 0,6 \\
\hline
\end{tabular}

*Proyección realizada de los datos correspondientes a los 7 días 


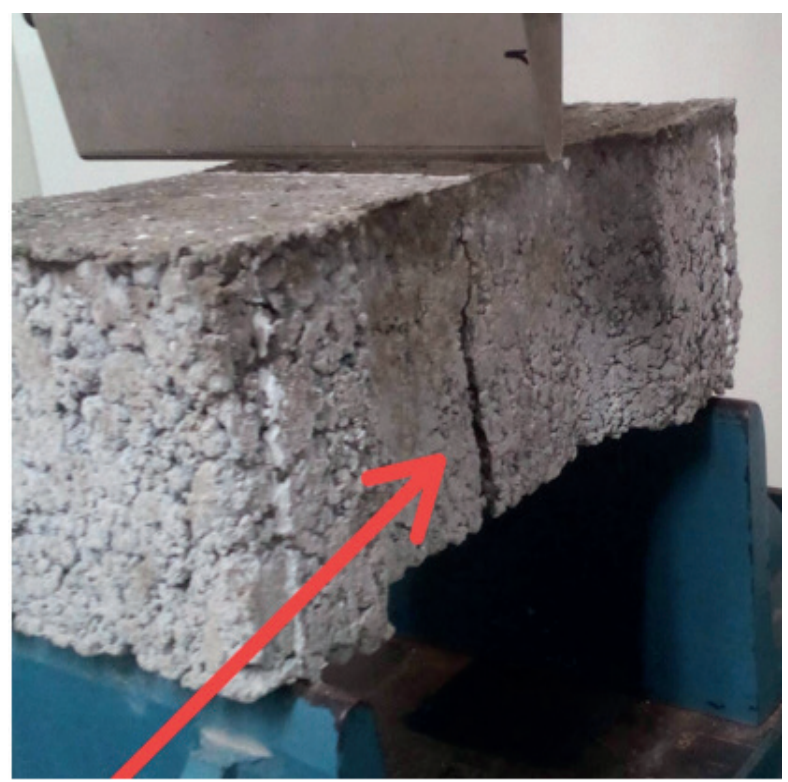

Figura 6. Rotura en la estructura del adoquín generada al alcanzar el esfuerzo máximo.

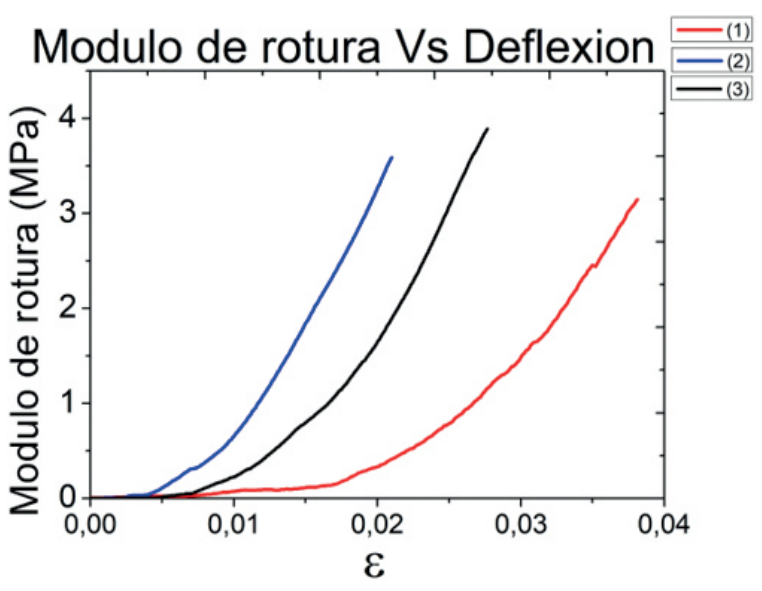

Figura 7. Módulo de rotura-deflexión de las muestras 1,2 y 3.

\section{Discusión}

Se consultó la norma técnica colombiana NTC 2017 para llevar a cabo las comparaciones respectivas, así se hallaron valores correspondientes al módulo de rotura mínimos presentes en los adoquines industriales (ver tabla 4).

El porcentaje de absorción, según la norma NTC 2017, no debe ser superior al 7\% (para todo el volumen del espécimen). Los adoquines obtenidos en este trabajo no superan dicho valor (ver tabla 2). El módulo de rotura (Mr) se ha especificado a los 28 días; sin embargo, los adoquines se pueden utilizar a edades más tempranas. Entendiendo que el Mr obtenido a los 7 días de curado corresponde a aproximadamente el $65 \%$ de su $\mathrm{Mr}$ máximo según Payá et al. [10] el cual es obtenido a los 28 días de curado. Por lo tanto se plantea una proyección del Mr de los adoquines obtenidos en este trabajo donde "28*" es la magnitud de $\mathrm{Mr}$ al realizar la proyección.

Se llevó a cabo una comparación de Mr promedio de tres muestras, (esta limitación se presentó debido a la disponibilidad de la máquina de ensayos, el tiempo durante el semestre para la realización de este proyecto y un accidente con dos de las muestras). La comparación se realizó entre los adoquines obtenidos en este trabajo y los valores admitidos por la NTC 2017. El resultado se consignó en la tabla 4. De esta tabla se puede observar que los Adoquines obtenidos en este trabajo presentan un valor de $\mathrm{Mr}$ en promedio los cuales, en un intervalo de confianza del $70 \%$, sí cumplen con las propiedades mínimas establecidas en la norma NTC 2017 con un promedio por encima del Mr especificado siendo esto una buena señal para una futura industria en torno al manejo de los RC\&D.

Tabla 4. Comparación entre los diferentes valores de Mr y absorción de agua.

\begin{tabular}{|c|c|c|c|c|}
\hline Muestra & $\begin{array}{c}\text { Mr a 28* días } \\
\text { (MPa) }\end{array}$ & $\begin{array}{c}\text { Mr mínimo NTC } 2018 \\
\text { (MPa) }\end{array}$ & Absorción de agua (\%) & $\begin{array}{c}\text { Máxima absorción } \\
\text { de agua permitida } \\
\text { NTC } 2018(\%)\end{array}$ \\
\hline 1 & 4,8 & 4,2 & 2,7 & 7,0 \\
\hline 2 & 5,5 & 4,2 & 2,4 & 7,0 \\
\hline 3 & 6,0 & 4,2 & 1,8 & 7,0 \\
\hline Promedio & 5,4 & 4,2 & 2,3 & 7,0 \\
\hline D. Estándar & 0,6 & 0,0 & 0,5 & 0,0 \\
\hline
\end{tabular}




\section{Conclusiones}

Los agregados obtenidos por medio de la trituración de escombros (morteros, ladrillos y concretos) poseen un buen desempeño para aplicaciones en adoquines, los cuales al llevarse a cabo el ensayo de flexotracción, arrojaron valores superiores a los establecidos por la norma técnica colombiana.

El uso de agregados obtenidos por medio de la conminución de RC\&D para nuevas aplicaciones en construcción, constituyen una alternativa que se debe considerar debido a su impacto ambiental a largo plazo si se constituye esta práctica como norma para la industria de la construcción.

El porcentaje de absorción presente en los adoquines con esta composición de agregados reciclados es bajo, no supera el valor máximo permitido por la NTC 2017, esto favorece a sus aplicaciones, por ejemplo, caminos peatonales en jardines para paisajismo arquitectónico.

\section{Recomendaciones}

Entendiendo que los RC\&D conllevan una dispersión considerable para los resultados de los ensayos físicos la cual varía en función de tamaño, naturaleza del escombro (ladrillo, mortero, hormigón) y forma de los mismos. Se recomienda realizar estudios con un mejor control de estos parámetros al realizar la mezcla, y de ser posible, contar con más especímenes al realizar los diversos ensayos físicos posibles. Además, considerar ensayos químicos para determinar también la respuesta ante la degradación de estos materiales reciclados.

\section{Agradecimientos}

A la Ing. Alexandra Moreno Rodríguez docente de gestión ambiental por su asesoramiento y motivación durante el curso "Introducción a la Gestión Ambiental". También a July Andrea Manquillo por su ayuda en el Laboratorio de Ensayos Físicos y Mecánicos de la Escuela de Ingeniería de Materiales de la Universidad del Valle.

\section{Referencias bibliográficas}

[1] Salazar RAR, Centeno PEM, Urrego YFS, Galindo DMB, y Arjona SD. Los residuos de la construcción y demolición en la ciudad de Cali: un análisis hacia su gestión, manejo y aprovechamiento. Revista Tecnura. 2015;19(44):157-170.

[2] Acevedo Sánchez CD. Evaluación de la factibilidad de obtención de argamasa a partir del aprovechamiento de ladrillo y mortero contenidos en escombros (tesis de maestría). Cali, Colombia: Universidad de la República; 2015.

[3] Morales Alpízar M, Villalta Flórez-Estrada M. Guía de manejo de escombros y otros residuos de la construcción. Costa Rica: UICN; 2012.

[4] Alcaldía de Santiago de Cali. Plan de Gestión Integral de Residuos Sólido de la ciudad de Santiago De Cali, Decreto Municipal 1147. Cali, Colombia; 2015.

[5] Ministerio de Ambiente y Desarrollo sostenible. Resolución 472. Bogotá, Colombia; 2017.

[6] Casa Editorial El País Cali, Los botaderos de escombros, otro problema que desborda a Cali. 2018.

[7] Zou G, Sun X, Liu X, Zhang J. Influence factors on using recycled concrete aggregate in foamed asphalt mixtures based on tensile strength and moisture resistance. Construction and Building Materials. 2020; 265: 120363. https://doi. org/10.1016/j.conbuildmat.2020.120363.

[8] Beja IA, Motta R, Bernucci LB. Application of recycled aggregates from construction and demolition waste with Portland cement and hydrated lime as pavement subbase in Brazil. Construction and Building Materials. 2020; 258: 119520. https://doi.org/10.1016/j. conbuildmat.2020.119520

[9] ICONTEC, (2004). NORMA TÉCNICA COLOMBIANA NTC 2017 segunda actualización. ADOQUINES DE CONCRETO PARA PAVIMENTOS / E: Concrete Paving Blocks.

[10] Valcuende Payá MO, Marco Serrano E, Jardón Giner R, Gil Andrés A. Alejandro. Evolución de la resistencia del hormigón con la edad y la temperatura (Tesis). Madrid, España: Universidad Politécnica de Valencia; 2011. 